This item was submitted to Loughborough's Research Repository by the author.

Items in Figshare are protected by copyright, with all rights reserved, unless otherwise indicated.

\title{
Effects of the turbulence model and the spray model on predictions of the n- heptane jet fuel-air mixing and the ignition characteristics with a reduced chemistry mechanism
}

\section{PLEASE CITE THE PUBLISHED VERSION}

https://doi.org/10.1177/0954407016687478

PUBLISHER

SAGE @ IMechE

VERSION

AM (Accepted Manuscript)

\section{PUBLISHER STATEMENT}

This work is made available according to the conditions of the Creative Commons Attribution-NonCommercialNoDerivatives 4.0 International (CC BY-NC-ND 4.0) licence. Full details of this licence are available at: https://creativecommons.org/licenses/by-nc-nd/4.0/

\section{LICENCE}

CC BY-NC-ND 4.0

\section{REPOSITORY RECORD}

Wei, Haiqiao, X. [Tianjin University] Chen, Wanhui Zhao, Lei Zhou, and Rui Chen. 2018. "Effects of the Turbulence Model and the Spray Model on Predictions of the N-heptane Jet Fuel-air Mixing and the Ignition Characteristics with a Reduced Chemistry Mechanism". figshare. https://hdl.handle.net/2134/28184. 


\title{
Effects of the turbulence model and the spray model on predictions of the $n$-heptane jet fuel-air mixing and the ignition characteristics with a reduced chemistry mechanism
}

\author{
HaiqiaoWei ${ }^{1}, X_{i}$ Chen $^{1}$, Wanhui Zhao $^{1}$, Lei Zhou ${ }^{1,{ }^{*}}$ and Rui Chen ${ }^{1,2}$ \\ ${ }^{1}$ State Key Laboratory of Engines, Tianjin University, Tianjin, People's Republic of China \\ ${ }^{2}$ Department of Aeronautical and Automotive Engineering, Loughborough University, Loughborough, \\ UK \\ *Corresponding author: lei.zhou@tju.edu.cn (Lei Zhou)
}

Accepted for publication by Proc IMechE Part D: J Automobile Engineering (2017)

\begin{abstract}
Reynolds-averaged Navier-Stokes simulations with an improved spray model and a realistic chemistry mechanism are performed for turbulent spray flames under diesel-like conditions in a constant-volume chamber. Comprehensive numerical analyses including two turbulence models (the renormalisation group $\mathrm{k}-\mathrm{e}$ model and the standard two-equation $k-\varepsilon$ model) with different model coefficients are made. The distribution of the fuel mixture fractions is a very important factor affecting the combustion process. In this study, we also use the entrainment gas-jet model, modifications of the the spray model coefficient and two turbulence models to investigate extensively the influence of the gas-jet theory model on the fuel-air mixture process. First, a nonreacting case is validated by comparing the liquid-phase penetration and the vapour-phase penetration and also the mixture fractions at different axis positions. Second, appropriate methods are confirmed according to accurate mixture fraction distributions to validate the combustion process. Because of the large number of species and reactions, the calculation of chemically reacting flows is unaffordable, particularly for three dimensional simulations. Hence, the dynamic adaptive chemistry method for efficient chemistry calculations is extended in this work to reduce the computational cost of the spray combustion process when a reduced chemistry mechanism is used. The results show that, in the evaporation case, the gas-jet theory model can be used to obtain a relatively accurate fuel vapour penetration length with different influential factors and that improved numerical methods can effectively reduce the mesh dependence for the spray evaporation process. It is demonstrated that the Schmidt number $S c$ and the turbulence models significantly influence the mixture fraction distribution. Very good agreement with available experimental data is found concerning the ignition delay time and the flame lift-off length for different oxygen concentrations owing to the accurate fuel mixture fraction.
\end{abstract}

\section{Keywords}

Mixture fraction, gas jet, spray, turbulence, dynamic adaptive chemistry 


\section{Introduction}

In order to meet increasingly stringent emissions regulations in the future and to improve the performance of combustion in internal-combustion engines, an effective and reasonable spray process needs to be achieved. The distribution of the fuel spray mixture in the combustion chamber has a very important factor influencing the combustion efficiency and the production of emissions for a diesel engine. ${ }^{1}$ At present, apart from experimental measurements, computational fluid dynamics (CFD) is also an effective measure to design and optimise the combustion system to form the best spray mixture. Thus, accurately predicting the fuel mixture fraction can effectively be utilised to obtain the parameters of the combustion process, such as the autoignition delay time. The objective of this study is to represent accurately the liquid spray and combustion characteristics using an improved spray model and a reduced chemistry mechanism.

In CFD, there are two different ways to simulate the liquid-phase flow and the gas-phase flow. The two methods are as follows: the Eulerian method, where both the liquid phase and the gas phase are considered as a continuum fluid in the whole flow domain; the Lagrangian method, where the liquid phase, which is described by clusters of droplets, is tracked through the whole flow domain. When the Eulerian method is used to describe the liquid phase, in general, the gas-liquid interface needs to be tracked to identify the location of the liquid phase, such as by the marker-andcell technique, ${ }^{2}$ the volume-of-fluid technique or the level set technique. This method can be used to obtain the liquid-gas flow structure with a high fidelity. However, with this method, the computational cost is extremely high, ${ }^{3}$ and it is only appropriate for the domain near the nozzle region. Thus, the Lagrangian-drop-Eulerian-fluid (LDEF) method has been widely used to model the gas-phase flow and the liquid-phase flow ${ }^{4}$ because of its lower cost, in which the liquid fuel is represented by a number of discrete particles tracked by the Lagrangian particle-tracking method, and the ambient fluid (gas) is treated as a continuum fluid solved by the Eulerian method. The twophase interaction is solved by adding spray source terms to the gas-phase conservation equations.

In this way, although the Lagrangian particle-tracking method can be used to obtain the trajectory of the motion of droplets with a high efficiency, it also demonstrates a strong grid dependence. ${ }^{5,6} \mathrm{~A}$ previous study found that this method may cause incorrect momentum coupling between the gas phase and the liquid phase and may result in resolution-dependent results when the spatial distribution of droplets is highly non-uniform. ${ }^{7}$ Abraham ${ }^{8}$ investigated the grid dependence of the spray models for vaporising diesel sprays in a constant-volume chamber and suggested that the nozzle region must be adequately resolved to obtain an accurate prediction of the spray structure. Later, Beard et al. ${ }^{6,9}$ reported that the relative velocity between the liquid phase and the gas phase is mesh dependent and thus may result in a lower axial velocity and incorrect liquid and vapour penetration lengths by comparison of the axial velocity distribution and the liquid and vapour penetration lengths for a vaporising diesel spray when using the LDEF method. They proposed gaseous particles and a sphere of momentum influence to modify the error relative velocity. This method gradually releases the vapour and momentum to mesh following a specified diffusion law. Subsequently, Sterno et al. ${ }^{10}$ also used the same concept to improve the numerical accuracy of a spray process in a diesel engine.

Another improved spray model based on the gas-jet theory ${ }^{11}$ was proposed ${ }^{12}$ to reduce the grid dependence. Lee and co-workers ${ }^{13,14}$ investigated the spray tip penetration of diesel sprays over 
wide ranges of ambient gas densities using an improved spray model based on the gas-jet theory. The results showed that the improved spray model based on the gas-jet theory can effectively increase the spray tip penetration. The improved model used by Kokjohn and Reitz ${ }^{15}$ was applied to the spray combustion process over a range of engine combustion regimes from non-premixed conditions to premixed conditions. Shuai et al. ${ }^{16}$ also used the improved model for low-temperature diesel combustion. Although the current improved spray model based on the gas-jet theory can be employed to obtain a good liquid penetration, there is less in-depth information on spray atomisation, evaporation and combustion, particularly the fraction distribution and the ignition of the mixture. In terms of the above analysis, the objective of this paper is to investigate the influencing factors of the distribution of the mixture fractions and the ignition in term of the improved spray model based on the gas-jet theory in the spray combustion process.

Chemistry mechanisms have been applied to the autoignition process and the reaction composition including emissions in diesel engine conditions. Chemistry mechanisms play important roles in the reduction in pollutant emissions and the improvement in the engine performance, particularly in homogeneous charge compression ignition and premixed charge compression ignition engine research, where combustion is a chemical-reaction-governed process. ${ }^{17}$ Thus, the importance of accurate chemistry mechanisms and their computational efficiencies is emphasised. Therefore, in this work, a reduced chemistry mechanism is used. Furthermore, in order to reduce the computation time of spray combustion process for three-dimensional simulations, a dynamic adaptive chemistry (DAC) methodology ${ }^{18,19}$ involving on-the-fly chemical mechanism reduction has demonstrated its ability to accelerate chemistry calculations.

Recently, efforts have been made to establish a reliable experimental database under engine-relevant conditions for the development and validation of the computational models, in which the Engine Combustion Network (ECN) ${ }^{20,21}$ provides a very wide range of measurements including the spray and combustion characteristics, which can be used to improve and validate numerical models. Many numerical studies ${ }^{22-27}$ of diesel spray turbulent combustion have been performed on the basis of experimental data to study the fundamental combustion issues and to validate the spray and combustion models for both Reynolds-averaged Navier-Stokes (RANS) simulations and largeeddy simulations (LESs). Bhattacharjee and Haworth ${ }^{27}$ attributed to the turbulence-combustion interactions in turbulent spray combustion, and in particular to low temperatures based on the RANS method with a probability density function (PDF). Pei et al. 22,23 simulated the diesel jet using RANS simulations with a PDF and LES with a $\delta$-function combustion model. The flame characteristics were well represented, and there were multiple ignition spots in the mixing layer. Tillou et al. ${ }^{24}$ performed a diesel spray combustion using two combustion models (the tabulated homogeneous reactor model with a PDF and the approximated diffusion flame presumed conditional moment model with a $\beta-P D F)$. Zhou et al..$^{25}$ addressed the spray process using the monotone upstream-centred schemes for conservation laws which can accurately represent the spray shape and the fuel vapour penetration based on the LES method.

The objective of this paper is to investigate the influencing factors in order to predict the spray characteristics in detail, particularly the fuel mixture fraction distribution, using a multidimensional computation code with an improved spray model based on the gas-jet theory and two turbulence models (the renormalisation group (RNG) $k-\varepsilon$ model and the standard two-equation $k-\varepsilon$ model). In previous studies, ${ }^{11-16}$ although the spray model based on the gas-jet theory can be used to 
obtain only good liquid penetration, in-depth information involving the distribution and the ignition of the fuel mixture has not been reported. Therefore, the important contribution of this work is that the performances of the improved spray model based on the gas-jet theory of the distributions of the mixture fractions and the ignition are demonstrated. Then, the performances of the above improved models are presented to simulate the spray combustion characteristics more accurately using the DAC method. In the study, the model validation is carried out using the data in the ECN. ${ }^{28}$

As an outline of the paper, fundamental numerical models including the spray models and the combustion approach and other computational models are reviewed in the second section. In the third section, the numerical conditions are introduced. Next, in the fourth section, the improved spray model is used in the evaporation process and the spray combustion process. Conclusions are presented in the fifth section.

\section{Methodology}

In the present study, the LDEF method is used to track the motion of the particles and to impose particle-fluid (gas) interactions. The improved spray model based on the gas-jet theory is used to reduce the grid dependence. The Kelvin-Helmholtz (KH) model and the Rayleigh-Taylor (RT) model, ${ }^{18}$ which have been widely used in recent literature, were implemented in the open-source KIVA code to predict the primary break-up and the subsequent secondary break-up. Moreover, the improved droplet collision mode ${ }^{29}$ is also used in these simulations. The CHEMKIN solver is also integrated into the KIVA-3V code for detailed chemistry calculations, and DAC ${ }^{26,30,31}$ is used to accelerate the chemistry calculations.

\section{The Lagrangian droplet equation}

The Lagrangian method, which is an efficient and accurate method for solving the spray dynamics, is based on the ideas of the Monte Carlo method and of the discrete-particle method. With these methods, the total droplet population is represented by a number of parcels, each of which consist of several physical droplets sharing the same sizes, positions, velocities and thermal properties. Thereafter, Lagrangian operation keeps track of the motion of these parcels by a set of ordinary differential equations, which solve the mass, momentum and energy exchange between the spray and the gas. Detailed information can be found in our previous work. ${ }^{32}$

\section{Gas-jet theory}

According to the previous studies, ${ }^{13,14}$ the relative velocity between the liquid phase and the gas phase is mesh dependent, and an improved spray model based on the gas-jet theory can efficiently reduce the mesh dependence of the LDEF method. Thus, the improved spray model is simply reviewed here.

In the equation for the magnitude of the relative velocity as shown below, $\tilde{u}_{i}$ is given as $\tilde{u}_{i}=$ $\left(\tilde{u}_{x}, \tilde{u}_{y}, \tilde{u}_{z}\right)$, where $\tilde{u}_{x}$ is the perpendicular component of the surrounding gas-phase velocity in the $x$ direction (and is obtained from the original computational solution), $\tilde{u}_{y}$ is the perpendicular component of the surrounding gas-phase velocity in the $y$ direction (and is also obtained from the original computational solution) and $\tilde{u}_{z}$ is the axial component which, in this work, is considered as 
in the $z$ direction (and is obtained from the entrained gas velocity model). At present, for the entrained gas velocity model according to the gas-jet theory, there are two equations (the steadystate equation and the unsteady-state equation) to calculate the entrained gas velocity $\tilde{u}_{z}$ as follows.

In the steady-state equation model, ${ }^{11} \tilde{u}_{z}$ is given by

$\tilde{u}_{z}=\min \left[U_{i n j}, \frac{3 U_{i n j}^{2} d_{e q}^{2}}{32 v_{t} z\left(1+3 U_{i n j}^{2} d_{e q}^{2} r^{2} / 256 v_{t} z^{2}\right)^{2}}\right]$

where $U_{i n j}$ is the injection velocity of the liquid jet, which is also assumed to be the injection velocity of the gas jet, $z$ is the axial distance of the droplet parcel from the nozzle and $r$ is the radial distance of the parcel from the spray axis. Thus, from the above equation, the relative velocity between the surrounding gas and the droplets in the near-nozzle region is assumed to be around zero. $d_{e q}$ is the equivalent diameter defined as $d_{e q}=d_{n o z} \sqrt{\rho_{l} / \rho_{g}}$, where $d_{n o z}$ is the effective nozzle diameter, $\rho_{l}$ is the density of the liquid phase and $\rho_{g}$ is the density of the gas phase. $v_{t}$ is the turbulent viscosity for jets given as $v_{t}=C_{t} \pi^{0.5} U_{i n j} d_{e q} / 2$, where $C_{t}$ is the entrainment constant and, in this work, $C_{t}=0.0161$ is selected.

In the unsteady-state equation model, ${ }^{33} \tilde{u}_{z}$ is given by

$\tilde{u}_{z}=\left[U_{i n j}, \frac{3 U_{i n j}^{2} d_{e q}^{2}}{K_{e n t r} Z} \frac{1}{\left(1+12 r^{2} / K_{e n t r}^{2} Z^{2}\right)^{2}}\right]$

where $K_{\text {entr }}$ is a model constant taken to be 0.7 , as suggested by Abani and Reitz. ${ }^{33}$ It should be noted that, in this work, the injection velocity is assumed to be a constant value and, in this equation, $U_{i n j}$ can be replaced by different initial velocities for various injection times.

\section{Break-up model}

The $\mathrm{KH}-\mathrm{RT}$ model ${ }^{34}$ is derived from the wave model, which is based on a linear stability analysis of liquid jets. The wave model sets the limit of stability and, if droplets exist for a sufficiently long time so that they become unstable, break-up occurs and the characteristics of the new droplets are based on the wavelength and the frequency of the instability that causes the break-up. Thus the break-up time and the break-up drop radius are defined as

$\tau=\frac{3.788 B_{1} r_{0}}{\Omega_{\text {wave }} \Lambda_{\text {wave }}}$

and

$r_{c}=B_{0} \Lambda_{\text {wave }}$

respectively, where $B_{0}$ and $B_{1}$ are empirical coefficients, $\Lambda_{\text {wave }}$ is the wavelength of the rapidly increasing wave and $\Omega_{\text {wave }}$ is the frequency of the rapidly increasing wave ( $\Lambda_{\text {wave }}$ and $\Omega_{\text {wave }}$ are obtained by curve fits from solutions of the dispersion equation that describes the $\mathrm{KH}$ instability which grows on the surface of a cylindrical liquid jet penetrating into a stationary incompressible gas). The rate of change in the droplet radius is expressed as

$\frac{d r}{d t}=\frac{r-r_{c}}{\tau}$ 
The wave model considers only the stripping process of the droplets due to the growth of $\mathrm{KH}$ instabilities on the droplet surface, which results from the relative velocity between the gas phase and the liquid phase, and is further enhanced by adding the RT model to account for sudden catastrophic break-up due to the deceleration of the droplets. In the RT model, the break-up time and the break-up drop radius are given by

$\tau_{R T}=\frac{C_{\tau}}{\Omega_{R T}}$

and

$r=\frac{C_{3, R T} \Lambda_{R T}}{2}$

respectively, where $C_{\tau}$ and $C_{3, R T}$ are constants, $\Lambda_{R T}$ is the wavelength of the rapidly increasing wave and $\Omega_{R T}$ is the frequency of the rapidly increasing wave. The new model is called the KH-RT model, in which both $\mathrm{KH}$ instabilities and RT instabilities are considered.

\section{Dynamic adaptive chemistry}

DAC can be achieved by using methods based on a directed relation graph (DRG), ${ }^{35}$ which are employed to eliminate as many unimportant species and related reactions as possible to accelerate the chemical step and simultaneously to maintain the prediction accuracy results compared with the detailed mechanism. In this study, we consider a reacting gas-phase mixture consisting of $n_{s}$ chemical species, composed of $n_{e}$ elements. The local thermochemical state of the mixture is completely characterised by the local pressure $p$, the sensible enthalpy $h_{s}$ of the local mixture and the vector $Y$ of the mass fractions of local species.

The DRG method is based on the observation that many species are only weakly coupled during the combustion process, such that the species that do not significantly affect the reaction rates of the major species can be eliminated from the mechanism. The first step of the DRG is to quantify species couplings by pairwise errors $r_{A, B}$ induced on a species $A$ by the elimination of another species $B$ for a given reaction state which consists of temperature and species concentrations; $r_{A, B}$ is given by

$r_{A, B}=\frac{\max _{i}\left|v_{A, i} \omega_{i} \delta_{B, i}\right|}{\max _{i}\left|v_{A, i} \omega_{i}\right|}$

where $\delta_{B, i}=1$ if the $i$ th reaction involves $B$ and, otherwise, $\delta_{B, i}=0, \omega_{i}$ is the net reaction rate of the ith reaction and $v_{A, i}$ is the stoichiometric coefficient of species $A$ in the $i$ th reaction.

\section{Numerical conditions}

The computational domain is set to be of cuboid geometry with a length of $30 \mathrm{~mm}$ and a height of $100 \mathrm{~mm}$. The three-dimensional grids employed have about $0.46 \times 10^{6}$ cells in total with the time step ranging from $1.0 \times 10^{-8} \mathrm{~s}$ to $1.0 \times 10^{-6} \mathrm{~s}$ depending on the physicochemical time scales and the Courant-Friedrichs-Lewy number in the simulations. A local dense grid which has about $0.46 \times 10^{6}$ cells, as shown in Figure 1 , is created by a modified K3PREP grid generator. No-slip 
adiabatic wall conditions are applied to all the boundaries except the injection section. To calculate the combustion process, an n-heptane reduced mechanism involving 94 species and 400 reactions including the 88 species and 387 reactions reported by Yoo et al. ${ }^{36}$ and the 13-step nitrogen oxide mechanism is used in this work. The well-stirred reactor model is employed in the combustion simulations. In order to accelerate the combustion computation, DAC ${ }^{26}$ based on the DRG-based methods $^{35}$ is used in this paper, which is employed to eliminate as many unimportant species and related reactions as possible to accelerate the chemical step and simultaneously to maintain the prediction accuracy compared with the detailed mechanism. Table 1 shows the main experimental conditions as reported by Pickett et al. ${ }^{1}$ The mixture fraction measurements were obtained with the Rayleigh-scattering method at long delays after the start of injection (ASOI). The Navier-Stokes equation is discretised using the finite volume method on an arbitrary hexahedral mesh by applying the arbitrary Lagrangian-Eulerian method. In this study, a quasi-second-order upwind differencing scheme is employed. The no-slip wall boundary condition is applied to all the boundaries of the computational domain. By considering the complex process for the break-up of the liquid fuel jet, the $\mathrm{KH}-\mathrm{RT}$ model ${ }^{37}$ is used to predict the primary break-up and the subsequent secondary droplet break-up. The collision and coalescence model used in this study is that reported by Nordin ${ }^{29}$ with the velocity interpolation model. Table 2 shows the important numerical methods and model coefficients used in this work. In Table 2 , the model coefficient $C_{\varepsilon 1}$ in the turbulent model is a parameter obtained by experience from the standard $k-\varepsilon$ model and a suitable value can be obtained for an accurate turbulent flow. The model coefficients $B_{1}$ and $C_{\tau}$ are also parameters obtained by experience from the $\mathrm{KH}-\mathrm{RT}$ model to determine the break-up time in the $\mathrm{KH}$ model and the RT model respectively. The model coefficient $K_{\text {entr }}$ is the parameter which was found by experience to influence the final gas velocity. Significant efforts have been devoted to the grid dependence of the spray process for liquid fuel based on the gas-jet theory in the previous studies, ${ }^{11-16}$ which has an important influence on the development of spray modelling. Most studies concentrated the liquid phase without considering evaporation and, in particular, the fuel mixture fraction. The turbulence model and other influencing factors for the spray evaporation and ignition process in terms of the gas-jet model have not been studied. Thus, as shown in Table 2, the two turbulence models with modified model coefficients based on the RANS method, the break-up model coefficient and the gas-jet model with modified model coefficients are employed in this work.
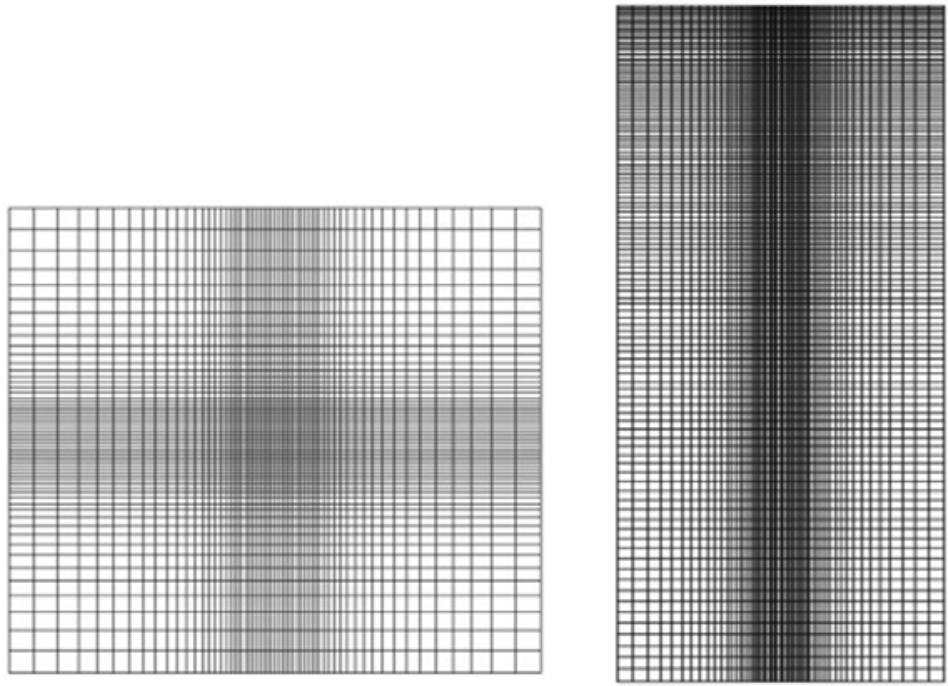

Figure 1 Computational mesh: top and midplane views. 
Table 1. Experimental conditions.

\begin{tabular}{ll}
\hline Parameter & Description or value \\
\hline Fuel & $\mathrm{C}_{7} \mathrm{H}_{16}$ \\
Diameter $(\mathrm{mm})$ of the hole & 0.1 \\
Injection duration $(\mathrm{ms})$ & 6.8 \\
Injection pressure $(\mathrm{MPa})$ & 150 \\
Mass $(\mathrm{mg})$ of the fuel & 17.8 \\
Temperature $(\mathrm{K})$ of the fuel & 373 \\
Ambient gas oxygen & $0 \%$ (non-reacting) \\
& $10-21 \%$ (reacting) \\
Ambient density $\left(\mathrm{kg} / \mathrm{m}^{3}\right)$ & 14.8 \\
Ambient temperature $(\mathrm{K})$ & 1000 \\
\hline
\end{tabular}

Table 2. The different numerical methods and various model coefficients.

\begin{tabular}{ll}
\hline Method & Coefficients \\
\hline Turbulence: standard & $C_{i: 1}=1.44$ (standard value in KIVA-3V) \\
& $C_{i .1}=1.52$ (improved) \\
& $C_{z 1}=1.6$ (baseline) \\
Turbulence: & Standard values in KIVA-3V \\
renormalisation group & \\
Break-up coefficients & $B_{1}=18.0, C_{\tau}=0.1$ (baseline) \\
& $B_{1}=40.0, C_{\tau}=1.0$ (test) \\
Gas-jet models & Steady-state model \\
& Unsteady-state model, $K_{\text {entr }}=0.7$ \\
& Unsteady-state model with a \\
& modified entrainment constant, \\
& $K_{\text {entr }}=0.45$ \\
\hline
\end{tabular}

\section{Results and discussion}

In fact, the previous studies mainly showed that the liquid penetration obtained by the gasjet method is independent of the computational grid. However, in this work, Figure $\mathbf{2}$ shows the distribution of the fuel vapour penetration lengths with and without the entrainment gas injection model based on the gas-jet theory using the RNG model. It can be found apparently that, with the entrainment gas injection model, the vapour penetration length can be increased owing to the enhanced gas-jet velocity. In terms of the comparison of the vapour penetration with the experimental data, a more detailed analysis presented as follows is based on a fine grid. Figure 3 shows the evolution of the fuel vapour penetration length using different methods involving the RNG turbulence method and model coefficients with a Schmidt number $S_{c}$ of 0.9 (the standard value in the KIVA code). When the gas-jet theory is considered, this means that the ambient gas velocity including the liquid droplet can be represented by a gas jet. The entrained air velocity is used to calculate more accurately the relative velocity between the gas phase and the droplets owing to a consistent gas-phase momentum with the ambient gas. It can be seen that penetration using the RNG model with the unsteady-state equation and the steady-state equation is in good agreement with the measured penetration data, particularly with the revised value of the break-up time coefficient from 0.1 to 1.0 and the revised value of the empirical constant $B_{1}$ from 18 to 40 . 


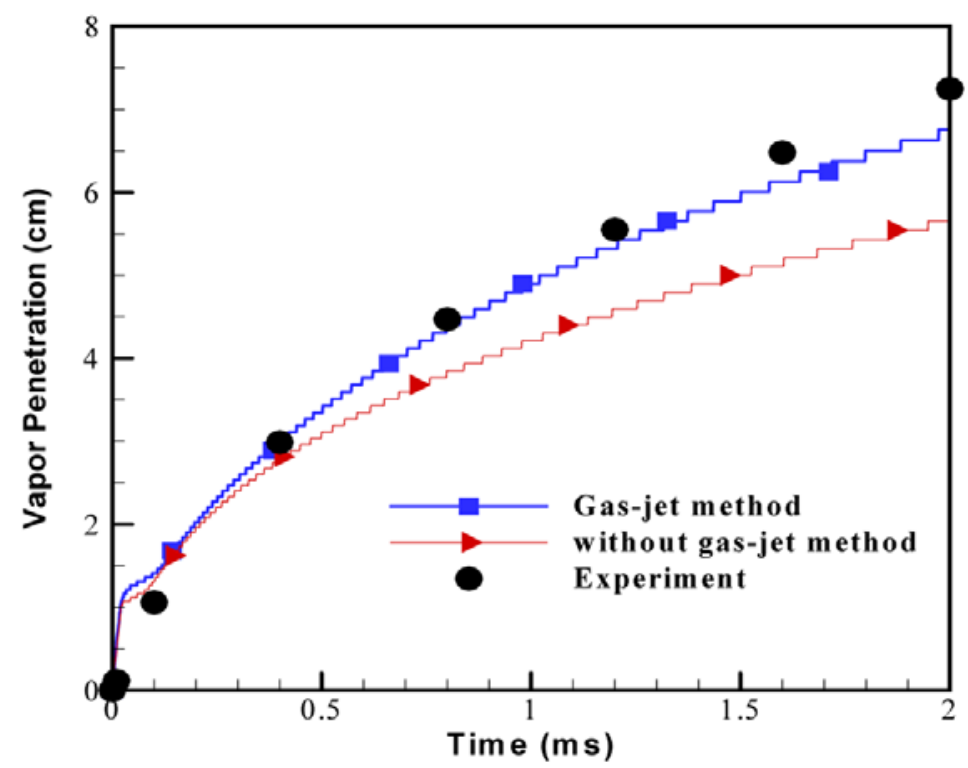

Figure 2 Distribution of the fuel vapour penetration lengths obtained by the gas-jet method compared with the result obtained without the gas-jet method based on the RNG turbulence model.

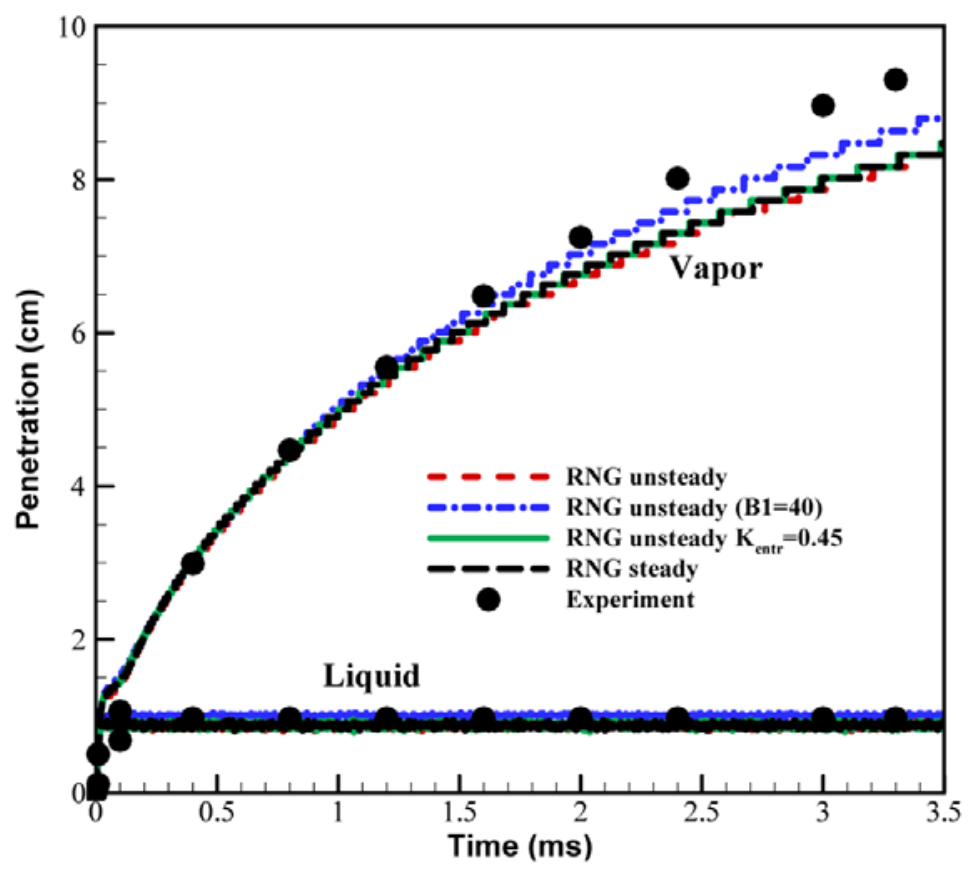

Figure 3 Evolution of the liquid penetration length and the fuel vapour penetration length obtained with different methods at $S C=0.9$. RNG: renormalisation group.

\section{Effect of diffusion}

For the spray flame, first, it is important to have an accurate computed mixture fraction distribution in the vicinity of the flame base field. Figure 4, Figure 5 and Figure 6 show the distributions of the mixture fractions at the three different positions $0.7 \mathrm{~cm}, 2.0 \mathrm{~cm}$ and $4.0 \mathrm{~cm}$ respectively for the different methods and model coefficients with $S c=0.9, S c=0.67$ and $S c=$ 0.5. The Schmidt number $S c$ is a dimensionless number defined as the ratio of the momentum 
diffusivity (viscosity) to the mass diffusivity $(S c=\mu / \rho D)$ and is used to characterise fluid flows in which there are simultaneous momentum and mass diffusion convection processes. As shown in Figure 4, none of the methods used in this work can be used to obtain an accurate mixture fraction at $1.7 \mathrm{~cm}$ at $0.49 \mathrm{~ms}$ ASOI with $S c=0.9$. When $S c$ is reduced from 0.9 to 0.67 , this means that a lower $S c$ value can increase the diffusion coefficient $D(=\mu /(\rho S c)$, where $\mu$ is the turbulent viscosity and $\rho$ is the density) according to the assumption of the Fick law in the present study, which can improve species diffusion. At $1.7 \mathrm{~cm}$, the gas-jet method can obtain a good mixing fraction distribution compared with the measured data with $S c=0.67$; however, around the injection axis it over-predicts the data. The steady-state equation can also be used to obtain similar good results, but they are slightly higher than the other results. However, as for the case with $S c=0.5$ in Figure 4 (c), the distribution of the mixture fractions is a little worse from $0.2 \mathrm{~cm}$ to $0.4 \mathrm{~cm}$. Like the results at 1.7 $\mathrm{cm}$, for the position at $2.0 \mathrm{~cm}$ at $6.0 \mathrm{~ms} \mathrm{ASOI}$, as shown in Figure 5, the methods with $S c=0.9$ fail to predict the distribution of the mixture fractions. With decreasing $S c$, the results improve, particularly for the unsteady-state equation with the revised $K_{\text {entr }}$ value of 0.45 . At $40 \mathrm{~mm}$, as shown in Figure 6, several methods have similar mixture fraction distributions for $S c=0.9$ and $S c=0.67$, except for the RNG turbulence model with the revised breakup model coefficient. At this position for $S c=0.5$, all the results are poor in comparison with the experimental data. Overall, the RNG turbulence model with the unsteady-state equation with $S c=0.67$ is in good agreement with the experimental results, in particular with the revised $K_{\text {entr }}$ value of 0.45 .

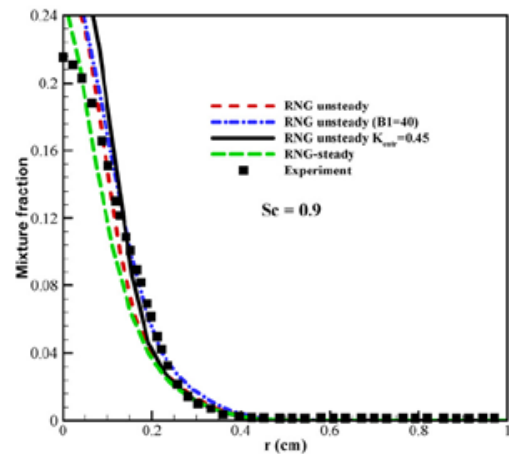

(a)

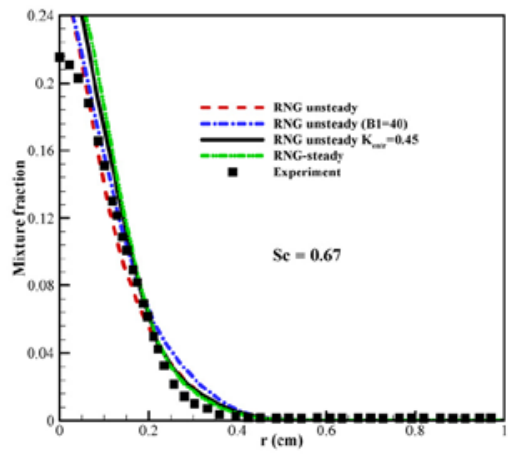

(b)

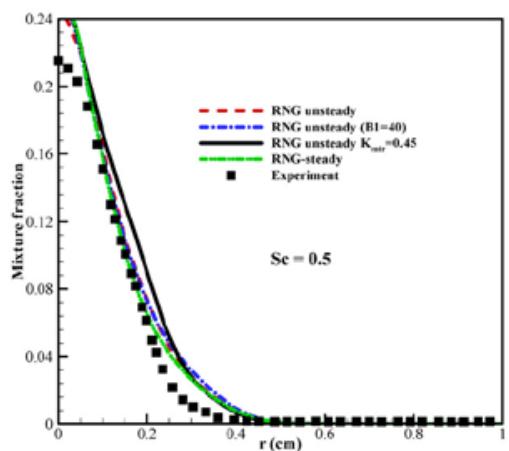

(c)

Figure 4 Mixture fractions at $1.7 \mathrm{~cm}$ at $0.49 \mathrm{~ms}$ ASOI with (a) $S c=0.9$, (b) $S c=0.67$ and (c) $S c=0.5$. RNG: renormalisation group.

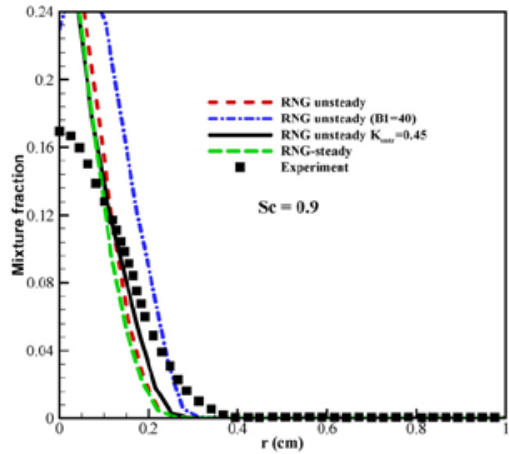

(a)

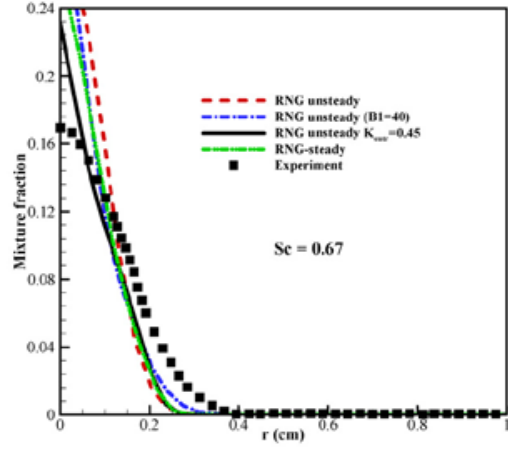

(b)

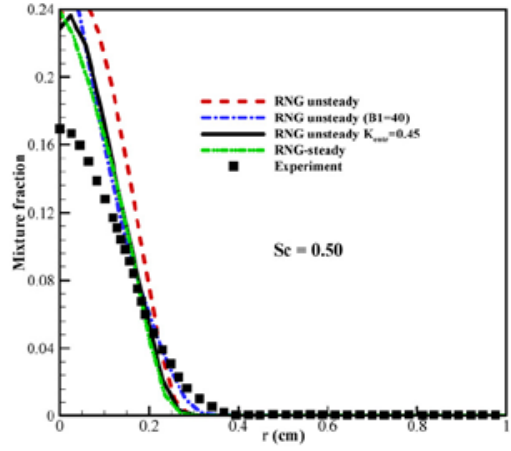

(c)

Figure 5 Mixture fractions at $2.0 \mathrm{~cm}$ at $6.0 \mathrm{~ms}$ ASOI with (a) $S c=0.9$, (b) $S c=0.67$ and (c) $S c=0.5$. RNG: renormalisation group. 


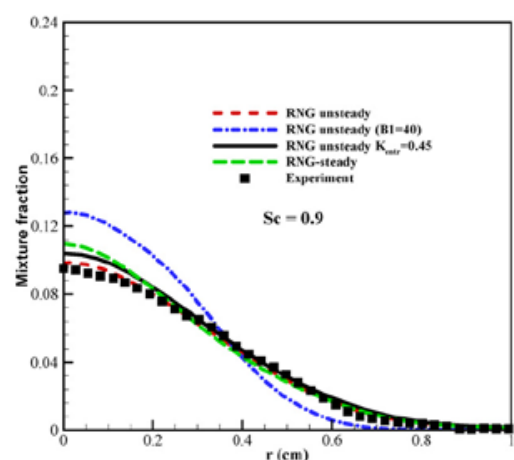

(a)

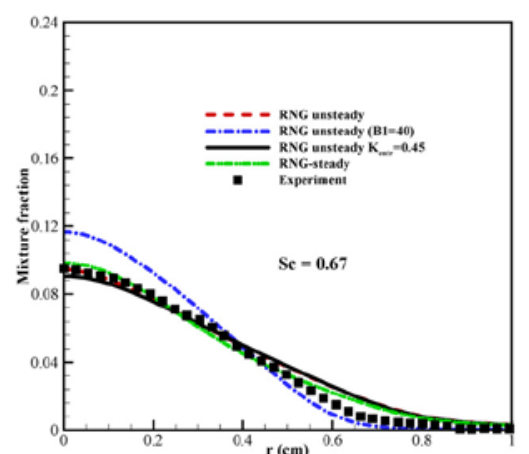

(b)

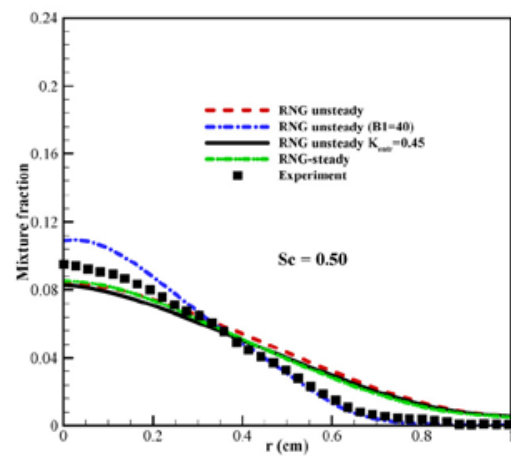

(c)

Figure 6 Mixture fractions at $4.0 \mathrm{~cm}$ at $6.0 \mathrm{~ms}$ ASOI with (a) $S c=0.9$, (b) $S c=0.67$ and (c) $S c=0.5$. RNG: renormalisation group.

Figure 7 shows the influence of the Schmidt number $S c$ on the liquid penetration length, the vapour penetration length and the mixture fraction on the spray axis line with the standard k-e turbulence model (the baseline case as shown in Table 2). It is evident that $S c$ does not have a great influence on the liquid penetration and the vapour penetration, although the mixture fraction distributions show an increasing trend with increasing $S c$. The peak value is around $1 \mathrm{~cm}$ from injector, which is the end position of the liquid position (the liquid penetration is about $1 \mathrm{~cm}$ ).

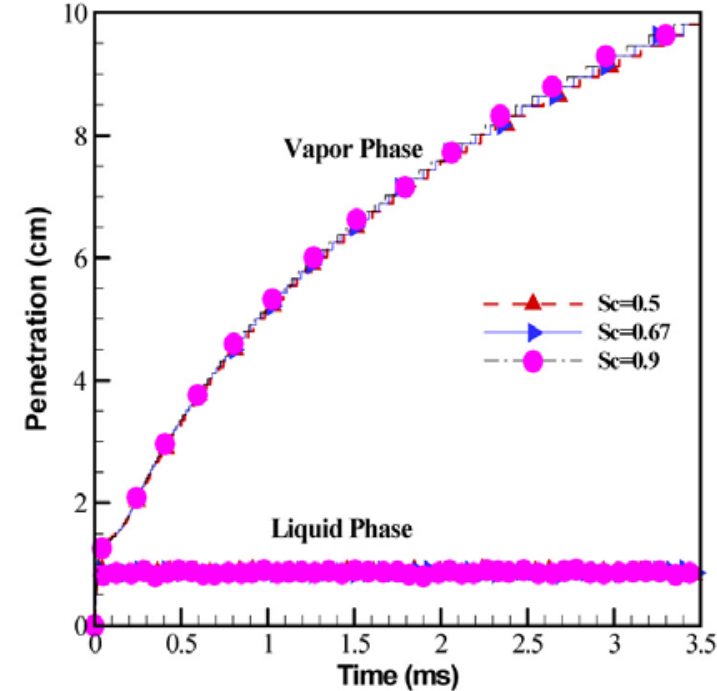

(a)

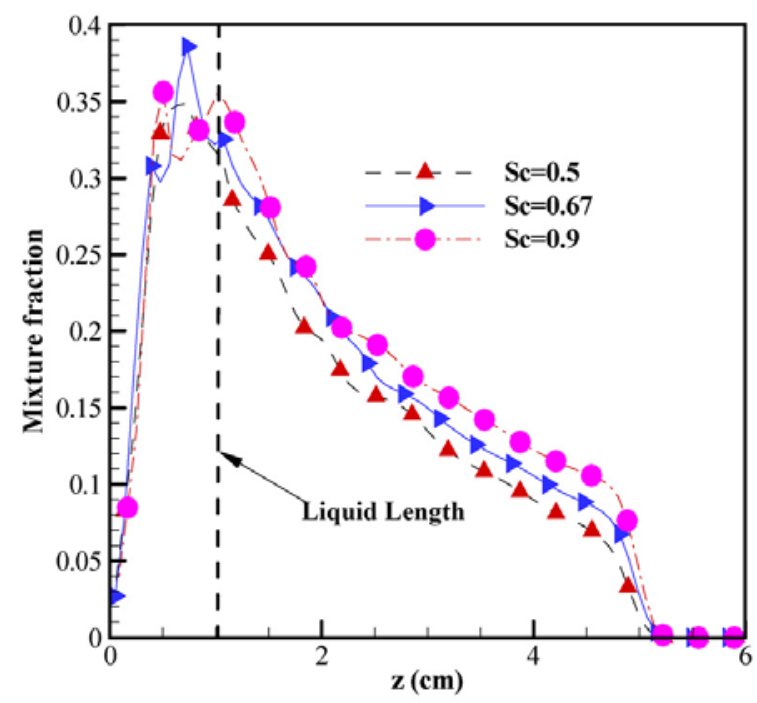

(b)

Figure 7 Curves of (a) the vapour penetration length versus the time and (b) the axial mixture fraction versus $z$ at $1.0 \mathrm{~ms}$ ASOI with $S c=0.5, S c=0.67$ and $S c=0.9$ using the standard turbulence model (the baseline as shown in Table 2).

\section{Effect of turbulence}

Figure 8 shows the evolution of the liquid penetration and the vapour penetration versus time and the axial mixture fraction distributions along the radial direction on the axial line with standard $k-\varepsilon$ turbulence model (baseline case) with different coefficients. For the value of 1.60 , the computed vapour penetration length is very close to the measured data for a long injection duration about $3.5 \mathrm{~ms} \mathrm{ASOI}$. For the other two values, the computed penetration is somewhat lower. As noted, the axial mixture fraction is reduced by decreasing the turbulence model coefficient in the 
same way as for the variation in the axial mixture fraction for different Schmidt numbers, as shown in Figure 7. Similar features can be found in the paper by James et al. ${ }^{38}$

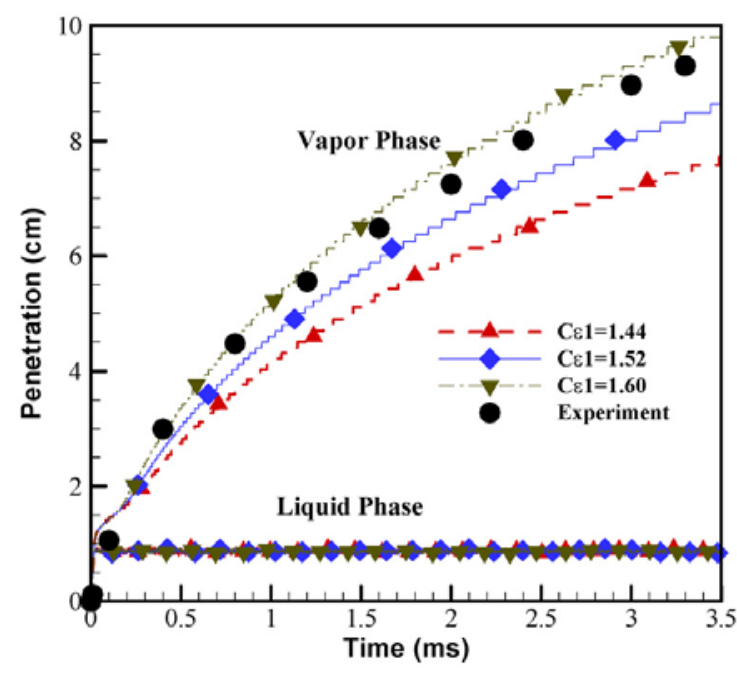

(a)

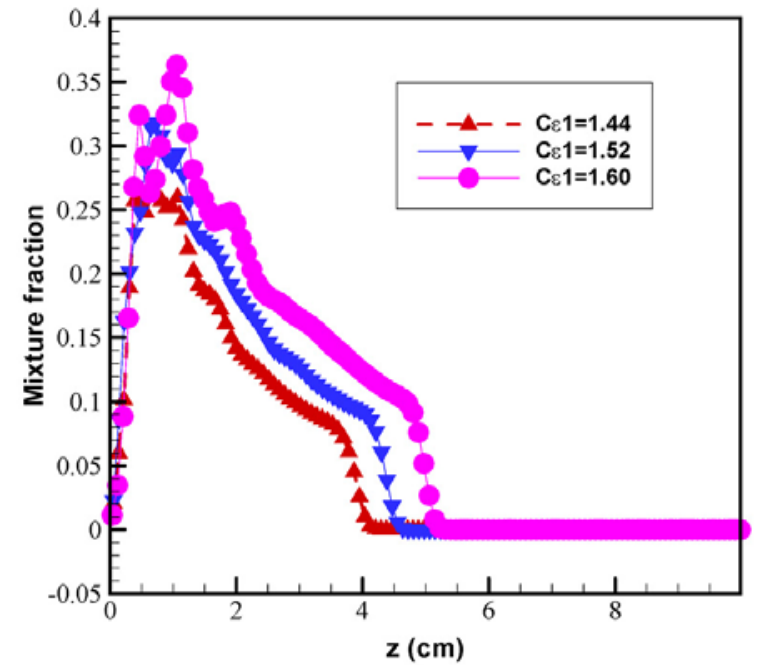

(b)

Figure 8 Curves of $(a)$ the vapour penetration length versus the time and (b) the axial mixture fraction versus $z$ at $1.0 \mathrm{~ms}$ ASOI with the standard $k-\varepsilon$ model coefficients $C_{\varepsilon 1}=1.44, C_{\varepsilon 1}=1.52$ and $C_{\varepsilon 1}=1.60$, using the unsteady-state equation.

Figure 9 shows the radial profiles of the mixture fractions with different turbulent coefficients and Schmidt numbers. It can be seen that, when $C_{\varepsilon 1}=1.60$ and $S c=0.5$, the mixture fraction distribution is close to the experimental data at the three radial positions. However, when the value of the turbulence model coefficient becomes 1.52, according to the above-mentioned variation trend of the mixture fraction following the turbulence model coefficient, the mixture fraction value becomes obviously lower than the experimental data, in particular at $1.7 \mathrm{~cm}$ at $0.49 \mathrm{~ms}$ ASOI in Figure 9(a) and at $4 \mathrm{~cm}$ at $6.0 \mathrm{~ms}$ ASOI in Figure 9(c). Thus, increasing $S c$ can increase the mixture fraction around the axial area and decrease the mixture fraction far from the axial area because of less diffusion. In regard to the mixture fraction distributions at the three positions, it is observed that calculation with $C_{\varepsilon 1}=1.52$ and $S c=0.9$ produces a relatively accurate spread rate of the species and is in reasonable agreement with the measured data.

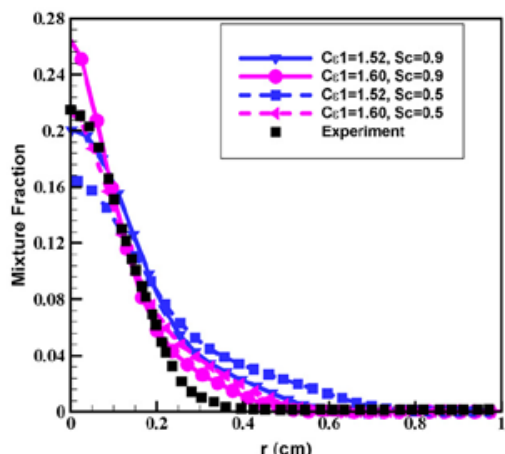

(a)

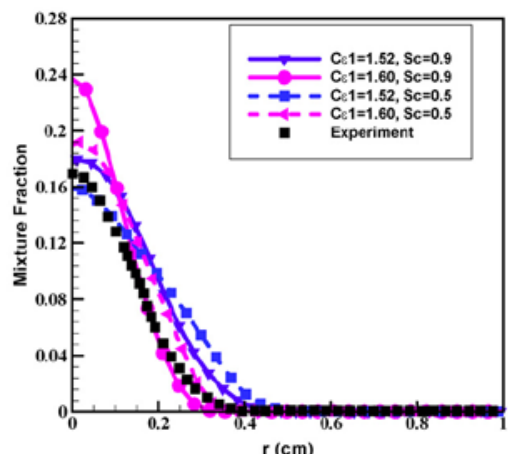

(b)

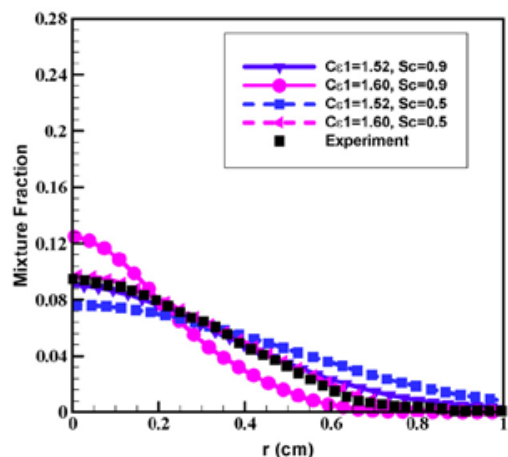

(c)

Figure 9 Mixture fractions at (a) $1.7 \mathrm{~cm}$ at $0.49 \mathrm{~ms} \mathrm{ASOl,} \mathrm{(b)} 2.0 \mathrm{~cm}$ at $6.0 \mathrm{~ms}$ ASOl and (c) $4.0 \mathrm{~cm}$ at $6.0 \mathrm{~ms}$ ASOI with the standard $k-\varepsilon$ model coefficients $C_{\varepsilon 1}=1.52$ and $C_{\varepsilon 1}=1.60$ and with $S c=0.9$ and $S c=0.5$, using the unsteady-state 


\section{Effect of the gas-jet theory}

Figure 10 shows the radial distributions of the mixture fractions with the unsteady-state equation, with the unsteady-state equation with the revised $K_{\text {entr }}$ value of 0.45 and with the steadystate equation using the standard turbulence model with the coefficient $C_{\varepsilon 1}=1.52$. As expected, the results are in excellent agreement with the data at all the three axial locations. On comparison of the results, the calculation results with the unsteady-state equation with the revised $K_{\text {entr }}=0.45$ are more accurate, particularly at $20 \mathrm{~mm}$. Thus, with regard to the standard turbulence $k-\varepsilon$ model, the spray methods can slightly adjust the mixture fraction distribution. Furthermore, the distributions of the fuel equivalence ratio using the standard turbulence $k-\varepsilon$ model with the coefficient $C_{\varepsilon 1}=1.52$ are shown in Figure 11. It can be seen that the present method appropriately reproduces the experimental trends in terms of the vapour penetration and distribution at different times in the entire domain.

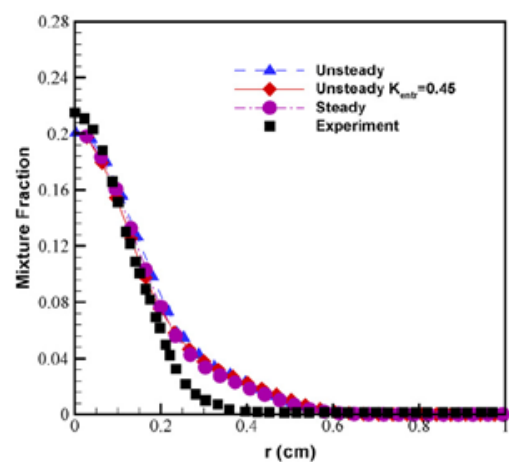

(a)

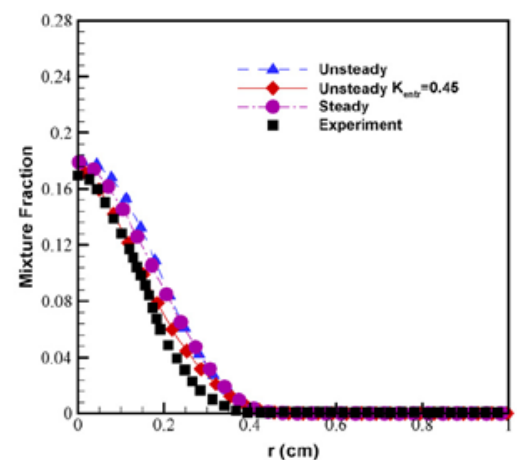

(b)

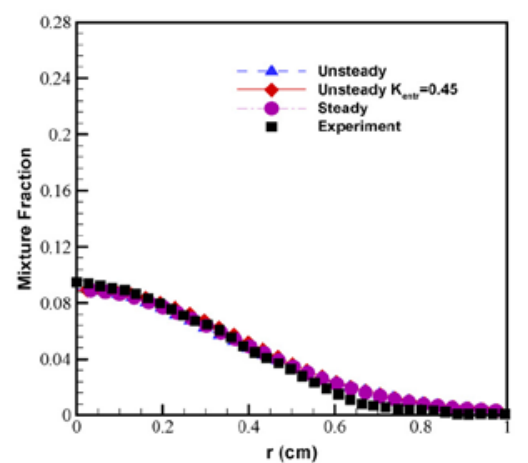

(c)

Figure 10 Mixture fractions at (a) $1.7 \mathrm{~cm}$ at $0.49 \mathrm{~ms}$ ASOI, (b) $2.0 \mathrm{~cm}$ at $6.0 \mathrm{~ms}$ ASOI and (c) $4.0 \mathrm{~cm}$ at $6.0 \mathrm{~ms}$ ASOI with the standard $k$-e model coefficients $C_{\varepsilon 1}=1.52$ using different methods
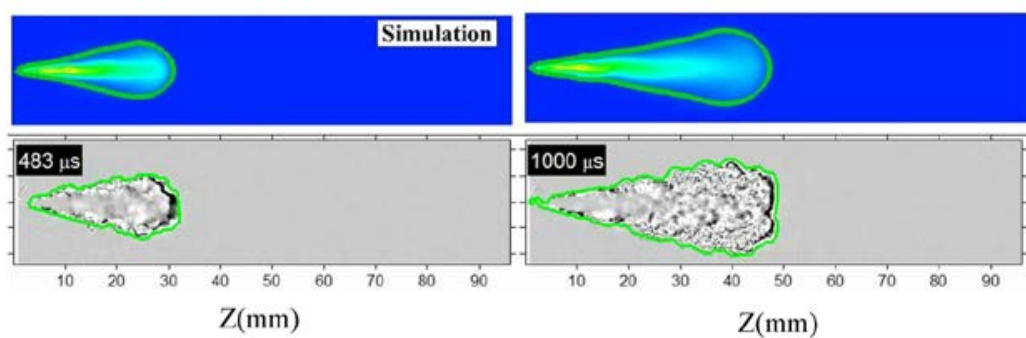

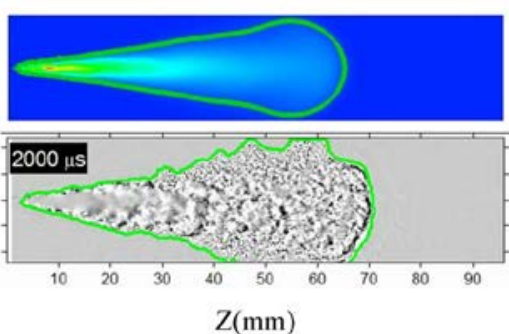

Figure 11 Equivalence ratio contour plots (top) with the standard $k-\varepsilon$ model coefficient $C_{\varepsilon 1}=1.52$ at 2.0 ms $A S O I$ at a temperature of $1000 \mathrm{~K}$ compared with the experimental data (bottom).

According to above studies, the RNG model with the revised Kentr value of 0.45 and $S c=$ 0.67 , and the standard model with $S c=0.5$ and $C_{\varepsilon 1}=1.6$ and the standard $k-\varepsilon$ model with $S c=$ 0.9 and $C_{\varepsilon 1}=1.52$ can be used to obtain the mixture fraction distributions at the three radial positions which agree relatively well with the measured data.

\section{Application to combustion}

Based on the above studies, the present method using the standard turbulence model with the coefficient $\mathrm{Ce} 1=1.52$ is employed to predict the combustion process including the ignition delay time and the flame lift-off length (LOL) for different oxygen concentrations qualitativey, as shown in Figure 12. In order to accelerate the chemistry calculation with a reduced chemistry mechanism, 
DAC with $\varepsilon_{D A C}=0.1$ is used in this work. In our previous study, ${ }^{26}$ the results have proved that using DAC with $\varepsilon_{D A C}=0.1$ can achieve an additional speed-up factor of about 2 without loss of accuracy. The LOL is defined at the location where the temperature reaches half the total temperature in the entire combustion process in the domain. ${ }^{39}$ It can be seen that the overall trends of the ignition delay time and the LOL are consistent with the experimental data. However, the present model over-predicts the ignition delay time since the turbulence-chemistry interaction at an oxygen concentration of $10 \%$ is not considered in this work. Zhou et al. ${ }^{26}$ showed that the turbulencechemistry interaction has an important effect only at low temperatures and low oxygen concentrations. It is noted that the chemistry mechanism also influences the ignition delay time at low temperatures and low oxygen concentrations significantly.

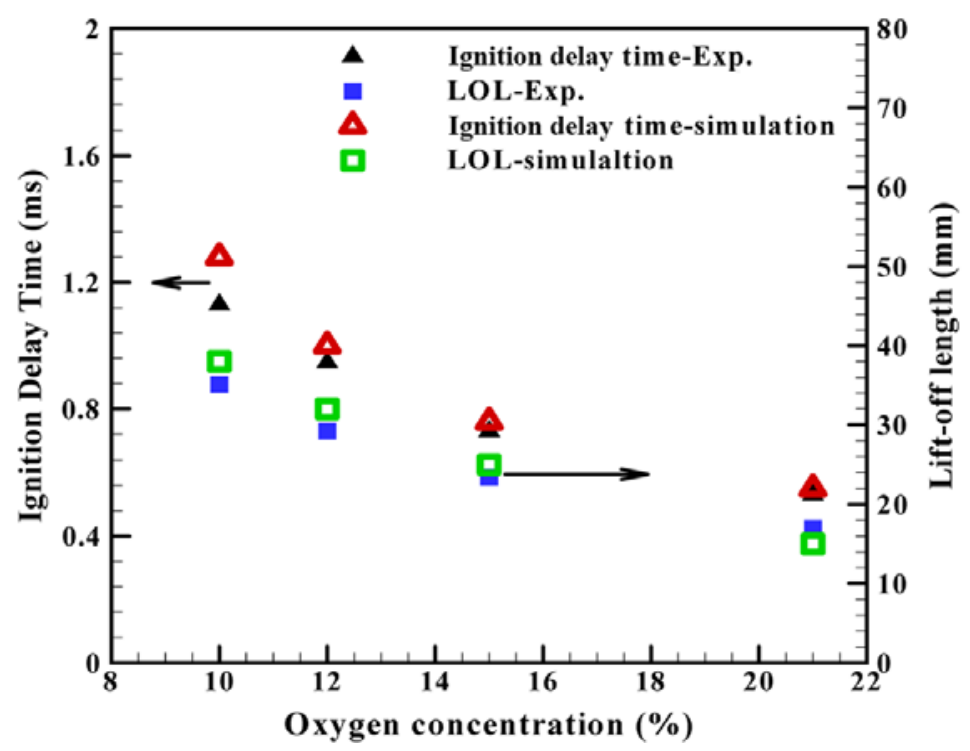

Figure 12 Flame LOL and ignition delay time using DAC with $\varepsilon_{D A C}=0.1$ for different oxygen concentrations compared with experimental data. Exp.: experiment; LOL: lift-off length.

Figure 13 shows the evolution of the fractions of globally retained species and reactions over the whole computational domain from the start of the injection. It should be noted that, at a given time instant, a species is counted as 'retained' if it is an active species, i.e. by DRG in the local skeletal mechanism in at least one CFD cell. As shown at the beginning of the simulations, the vaporisation and the mixing processes are dominant, where chemical reactions are weak and insignificant. With the evolution of the combustion process, the reaction activities are intensified, and more species and reactions become important. It can be seen clearly from the evolution of the retained species and the important intermediate species $\mathrm{CO}$ and $\mathrm{OH}$ that the rapid increase in the CO species is consistent with an increase in the retained species, which can represent the start of the initial heat release stage with a low-combustion process at the two oxygen concentrations of $21 \%$ and $12 \%$. After autoignition timing, the retained species then starts to increase. In the same way as the initial release stage, the autoignition timing is close to the time for producing an $\mathrm{OH}$ radical with a high temperature heat release. Figure $\mathbf{1 4}$ shows the map of the equivalence ratio and the temperature according to the number of retained species at $2.0 \mathrm{~ms}$ ASOI. It can be observed that the outer diffusion flame of the flame structure with a high temperature requires fewer species in the chemistry mechanism to describe the reaction process than does the inner structure region with a relatively low temperature. 


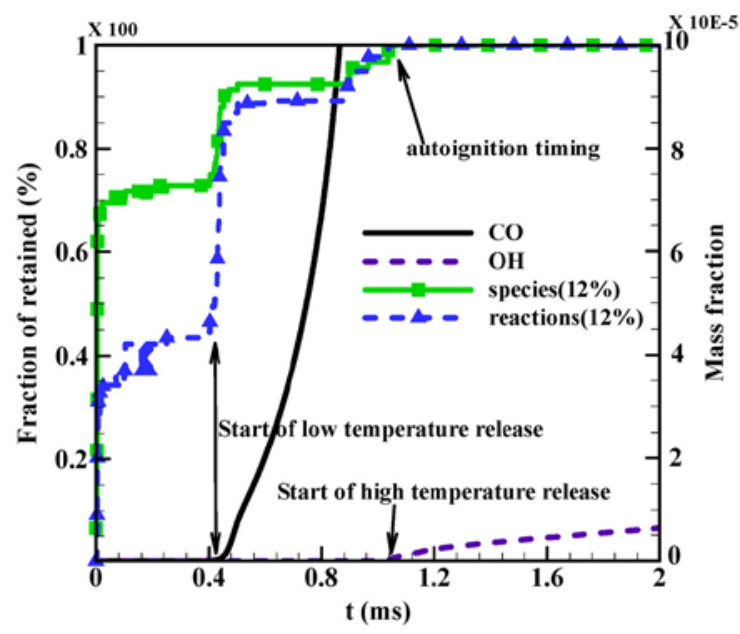

(a)

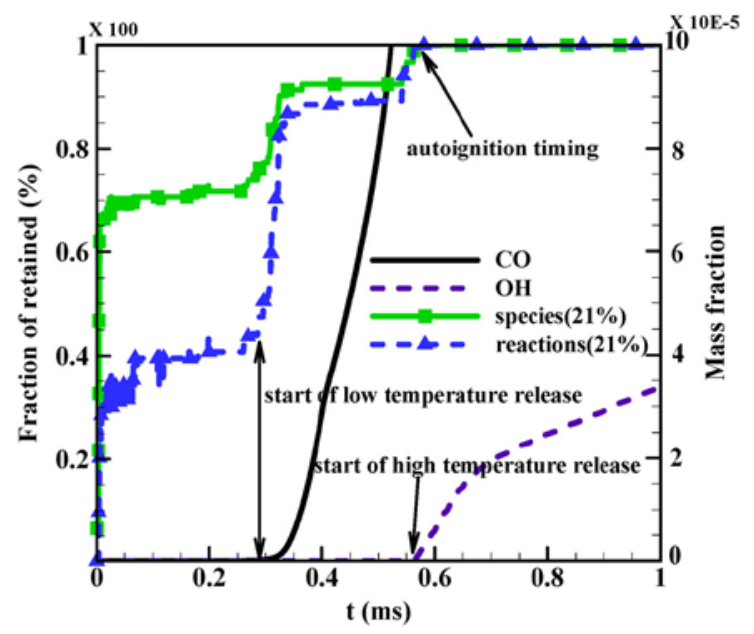

(b)

Figure 13 Evolution of the fractions of retained species and reactions in the mechanism with time for DAC at oxygen concentrations of (a) $12 \%$ and (b) $21 \%$.

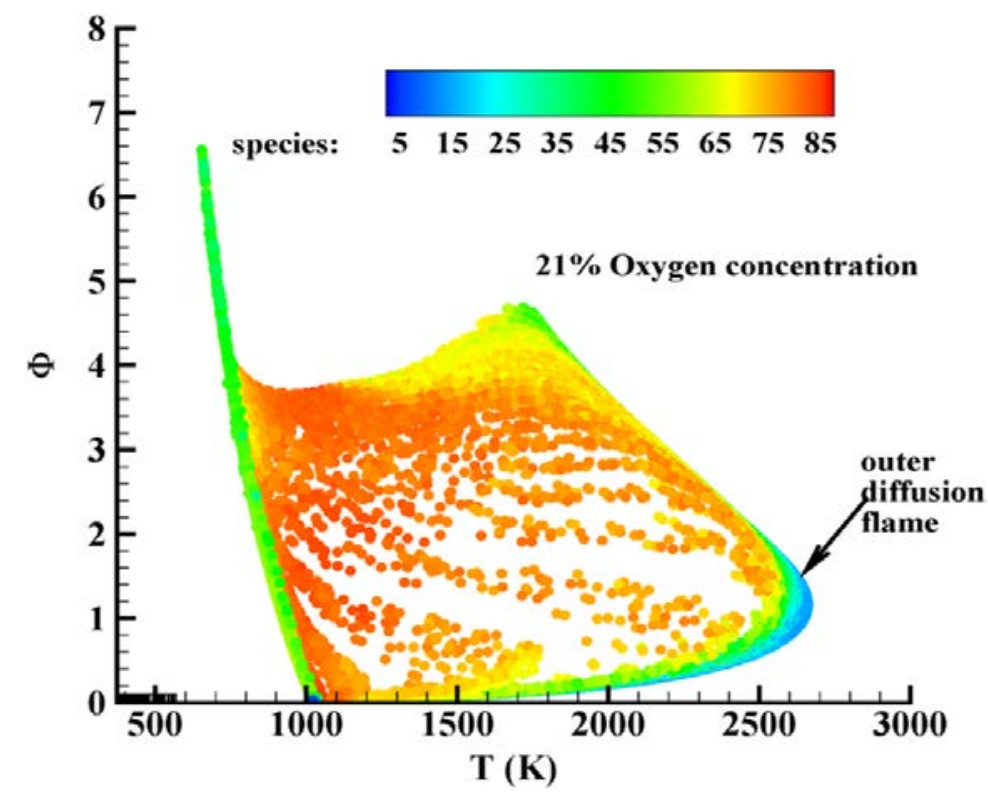

Figure 14 Space distributions of the numbers of retained species versus temperature where the various grey shades indicate the equivalent ratio for an oxygen concentration of $21 \%$ at $t=2.0 \mathrm{~ms} \mathrm{ASOI}\left(\varepsilon_{D A C}=0.1\right)$.

\section{Conclusion}

In this work, a parameter study is carried out to investigate the effects of the turbulence model and the spray model on predictions of the $n$-heptane jet fuel-air mixing and the ignition characteristics with a reduced chemistry mechanism. In order to describe accurately the gas-liquid interaction in the spray process, the entrainment gas injection models with different model parameters are employed to perform non-reacting and reacting spray combustion simulations. Specifically, because of the very different performance of the turbulence model for the mixture fraction distribution, the RNG model and the standard $k-\varepsilon$ turbulence model are tested. Furthermore, the DAC method with $\varepsilon_{D A C}=0.1$ and a reduced chemistry mechanism for $\mathrm{n}$-heptane 
(92 species) are used in this work. The ECN data for $n$-heptane are used to validate the present models in a constant-volume combustion chamber.

By carefully investigating the performances of the turbulence model and the spray model for the mixture fraction distribution, it can be concluded, by using three values of $S c$, that $S c$ does not have a great influence on liquid penetration and vapour penetration, although increasing Sc can increase the mixture fraction around the axial area and decrease the mixture fraction far from the axial area owing to less diffusion of the species. The axial mixture fraction is reduced by decreasing the turbulence model coefficient $C_{\varepsilon 1}$ for the standard $k-\varepsilon$ model in the same way as for the variation in the axial mixture fraction for different $S c$ values. The RNG model with the revised $K_{\text {entr }}$ value of 0.45 and $S c=0.67$, the standard model with $S c=0.5$ and $C_{\varepsilon 1}=1.6$ and the standard $k-\varepsilon$ model with $S c=0.9$ and $C_{\varepsilon 1}=1.52$ can be used to obtain mixture fraction distributions at three radial positions which agree relatively well with the measured data.

Based on studies of the non-reacting spray process, the present method using the standard turbulence model with the coefficient $C_{\varepsilon 1}=1.52$ is employed to predict the combustion process including the ignition delay time and the flame LOL for different oxygen concentrations qualitatively. The results show that the overall trends of the ignition delay time and the LOL using the present models are consistent with the experimental data. For the DAC method, it can be seen clearly from the evolution of the retained species and the important intermediate species $\mathrm{CO}$ and $\mathrm{OH}$ that the rapid increases in the $\mathrm{CO}$ and $\mathrm{OH}$ species are consistent with different heat release stages. Furthermore, in this work, the turbulent combustion model is not the objective of this work and is thus not considered. Therefore, in further work, the performance of the turbulent combustion model in spray combustion needs to be studied. It should also be noted that the previous studies have demonstrated that the spray model without the gas-jet theory based on LES also can be utilised instead of the present spray models to obtained an accurate spray penetration. However, the mixture fraction distribution predicted by LES is more complex and needs many more studies in future.

\section{Declaration of conflicting interests}

The author(s) declared no potential conflicts of interest with respect to the research, authorship, and/or publication of this article.

\section{Funding}

The author(s) disclosed receipt of the following financial support for the research, authorship, and/or publication of this article: This work was supported by the National Natural Science Foundation of China (Grant Number 51606133).

\section{References}

1. Pickett LM, Manin J, Genzale CL et al. Relationship between diesel fuel spray vapor penetration/dispersion and local fuel mixture fraction. SAE paper 2011-01-0686, 2011. 
2. Harlow FH and Welch JE. Numerical calculation of time-dependent viscous incompressible flow of fluid with free surface. Phys Fluids 1965; 8: 2182-2190.

3. Shinjo $J$ and Umemura A. Simulation of liquid jet primary breakup: dynamics of ligament and droplet formation. Int J Multiphase Flow 2010; 36: 513-532.

4. Jiang $X$, Siamas GA, Jagus $K$ and Karayiannis TG. Physical modelling and advanced simulations of gasliquid two-phase jet flows in atomization and sprays. Prog Energy Combust 2010; 36: 131-167.

5. Schmidt DP and Rutland C. A new droplet collision algorithm. J Comput Phys 2000; 164: 62-80.

6. Beard P, Duclos J-M, Habchi C et al. Extension of Lagrangian-Eulerian spray modeling: application to high-pressure evaporating diesel sprays. SAE paper 2000-01-1893, 2000.

7. Post $\mathrm{S}$, Iver $\mathrm{V}$ and Abraham J. A study of near-field entrainment in gas jets and sprays under diesel conditions. J Fluids Engng 2000; 122: 385-395.

8. Abraham J. What is adequate resolution in the numerical computations of transient jets? SAE paper 970051, 1997.

9. Beard $\mathrm{P}$, Colin $\mathrm{O}$ and Miche $\mathrm{M}$. Improved modelling of $\mathrm{DI}$ diesel engines using sub-grid descriptions of spray and combustion. SAE paper 2003-01-0008, 2003.

10. Sterno N, Greeves G, Tullis $S$ et al. Improvements of the KIVA dense spray modeling for HSDI diesel engines. SAE paper 2007-01-0001, 2007.

11. Abraham J. Entrapment Characteristics of transient gas jets. Numer Heat Transfer, Part A Applic 1996; 30: 347-364.

12. Abani N, Munnannur A and Reitz RD. Reduction of numerical parameter dependencies in diesel spray models. Trans ASME, J Engng Gas Turbines Power 2008; 130:032809.

13. Lee $\mathrm{CH}$, Wang $\mathrm{Y}$ and Reitz RD. CFD simulation of diesel sprays over a wide range of ambient gas densities using an improved gas jet spray model. Atomization Sprays 2011; 21: 591-609.

14. Lee $\mathrm{CH}$ and Reitz RD. CFD simulations of diesel spray tip penetration with multiple injections and with engine compression ratios up to 100: 1. Fuel 2013; 111: 289-297.

15. Kokjohn SL and Reitz RD. Investigation of the roles of flame propagation, turbulent mixing, and volumetric heat release in conventional and low temperature diesel combustion. Trans ASME, J Engng Gas Turbines Power 2011; 133: 1-10.

16. Shuai S, Abani N, Yoshikawa $T$ et al. Evaluation of the effects of injection timing and rate-shape on diesel low temperature combustion using advanced CFD modeling. Fuel 2009; 88: 1235-1244.

17. Ra $Y$ and Reitz RD. A combustion model for IC engine combustion simulations with multicomponent fuels. Combust Flame 2011; 158: 69-90. 
18. Lu Z, Zhou L, Ren Z et al. Effects of spray and turbulence modelling on the mixing and combustion characteristics of an $n$-heptane spray flame simulated with dynamic adaptive chemistry. Flow, Turbulence Combust 2016; 97:609-629.

19. Zhou $\mathrm{L}$ and Wei $\mathrm{H}$. Chemistry acceleration with tabulated dynamic adaptive chemistry in a realistic engine with a primary reference fuel. Fuel 2016; 171: 186-194.

20. Idicheria CA and Pickett LM. SAE paper 2007-01-0647, 2007.

21. Pickett LM, Manin J, Genzale $\mathrm{CL}$ et al. Relationship between diesel fuel spray vapor penetration/dispersion and local fuel mixture fraction. SAE paper 2011-01-0686, 2011.

22. Pei $Y$, Som S, Pomraning $E$ et al. Large eddy simulation of a reacting spray flame with multiple realizations under compression ignition engine conditions. Combust Flame 2015; 162: 4442-4455.

23. Pei $Y$, Hawkes ER and Kook S. Transported probability density function modelling of the vapour phase of an nheptane jet at diesel engine conditions. Proc Combust Inst 2013; 34: 3039-3047.

24. Tillou J, Michel JB, Angelberger C and Veynante D. Assessing LES models based on tabulated chemistry for the simulation of diesel spray combustion. Combust Flame 2014; 161: 525-540.

25. Zhou L, Luo KH, Qin W et al. Large eddy simulation of spray and combustion characteristics with realistic chemistry and high-order numerical scheme under diesel engine-like conditions. Energy Conversion Managmt 2015; 93: 377-387.

26. Zhou L, Lu Z, Ren Z et al. Numerical analysis of ignition and flame stabilization in an n-heptane spray flame. Int J Heat Mass Transfer 2015; 88: 656-571.

27. Bhattacharjee $S$ and Haworth DC. Simulations of transient $n$-heptane and $n$-dodecane spray flames under enginerelevant conditions using a transported PDF method. Combust Flame 2013; 160: 2083-2102.

28. Pickett LM. Engine combustion network, https://ecn.sandia.gov/ (2016, accessed 22 September 2016).

29. Nordin N. Complex chemistry modeling of diesel spray combustion. PhD Thesis, Chalmers University of Technology, Gothenburg, Sweden, 2001.

30. Ren Z, Liu Y, Lu T et al. The use of dynamic adaptive chemistry and tabulation in reactive flow simulations. Combust Flame 2014; 161: 127-137.

31. Yang H, Ren Z, Lu T and Goldin GM. Dynamic adaptive chemistry for turbulent flame simulations. Combust Theory Modelling 2013; 17: 167-183.

32. Zhou L, Luo KH, Shuai SJ and Xie MZ. Large-eddy simulations of diesel spray with a fine grid in a constantvolume vessel. Proc IMechE Part D: J Automobile Engineering 2014; 229(2): 247-260.

33. Abani N and Reitz RD. Unsteady turbulent round jets and vortex motion. Phys Fluids 2007; 19: 125102. 
34. Reitz RD. Modeling atomization processes in high-pressure vaporizing sprays. Atomisation Spray Technol 1987; 3: 309-337.

35. Lu T and Law CK. A directed relation graph method for mechanism reduction. Proc Combust Inst 2005; 30: 1333-1341.

36. Yoo CS, Lu T, Chen JH and Law CK. Direct numerical simulations of ignition of a lean $n$ heptane/air mixture with temperature inhomogeneities at constant volume: parametric study. Combust Flame 2011; 158: 1727-1741.

37. Patterson MA and Reitz RD, Modeling the effects of fuel spray characteristics on diesel engine combustion and emission. SAE paper 980131, 1998.

38. James $S$, Pope $S$, Anand $M$ and Razdan $M$. In situ detailed chemistry calculations in combustor flow analyses. Trans ASME, J Engng Gas Turbines Power 2001; 123: 747-756.

39. Bekdemir C, Somers LMT, de Goey LPH et al. Predicting diesel combustion characteristics with large-eddy simulations including tabulated chemical kinetics. Proc Combust Inst 2013; 34: 30673074. 